\title{
Le Visage de la Victoire di Henry De Groux: la riscoperta di un'opera antimilitarista del tempo della Prima guerra mondiale
}

Il presente intervento è dedicato a un'importante raccolta di incisioni, oggi riscoperta: Le Visage de la Victoire, eseguita dall'artista belga Henry De Groux. Costituita da oltre 40 incisioni (acqueforti e vernici molli), è stata realizzata nel corso della Prima Guerra Mondiale al fine di denunciare l'inutile massacro in corso. Si tratta di opere di ispirazione visionaria, che rivelano significati universali, mettendo il maestro belga in rapporto di affinità con artisti quali Goya e Otto Dix.

This article is dedicated to an important set of engravings, newly rediscovered: Le Visage de la Victoire, executed by the Belgian artist Henry De Groux. It consists of more than 40 prints (etchings and soft-ground etchings), realized during the WWI in order to denounce the needless slaughter in progress. These works, often of visionary inspiration, seem to uncover universal meanings, putting the Belgian master in a relationship of affinity with artists such as Goya and Otto Dix.

A partire dall'assassinio di Francesco Ferdinando a Sarajevo, i media di gran parte dei paesi europei si sono cimentati con zelo ineguagliabile nella prima grande opera di persuasione di massa intorno alla necessità di una guerra che necessaria probabilmente non era affatto. Instancabile fu allora il lavoro di artisti, scrittori, politici e giornalisti, rivolto a demonizzare l'avversario e a forgiare quella sorta di mitologia positiva della Prima guerra mondiale che (nonostante i milioni di morti, nonostante le immani distruzioni, nonostante gli orrori delle trincee e l'uso indiscriminato di armi di sproporzionata potenza e dei gas nervini) perdura in parte ancora oggi. In tale contesto, si annoverano pochi casi di artisti che hanno rappresentato la guerra per denunciarne la crudeltà e l'insensatezza, ancor più tra quelli che risiedevano nei paesi belligeranti. 
In questo quadro assai poco variegato, è assai peculiare la posizione sovversiva del maestro belga Henry De Groux (Saint-Josse-ten-Noode 1866 - Marsiglia 1930) che, operando a Parigi, in stretto contatto con i luoghi dei combattimenti, ha messo a nudo le dinamiche perverse che determinarono gli eventi bellici e le loro gravissime conseguenze. De Groux si recò anche al fronte, «per vedere i campi di battaglia, i soldati, i prigionieri, i rifugiati, i villaggi distrutti», come riferisce il genero Emil Baumann nella biografia a lui dedicata [Baumann 1936, 240]. La produzione realizzata dall'artista nel corso della guerra comprende oltre trecento opere, tra cui disegni incisioni e dipinti. Lavori che ancora oggi sono poco valorizzati, essendo in parte dispersi e in parte poco conosciuti. L'opera nella quale traspare in modo più strutturato il suo programma anti-militarista è la raccolta di quarantadue incisioni, intitolata Le Visage de la Victoire, realizzata tra l'agosto del 1914 e il novembre del 1916 [Morganti 2013]. Esposta la prima volta a Parigi alla Galerie Boëtie, nel dicembre del 1916, insieme con litografie e disegni inerenti gli stessi temi, la raccolta venne pubblicata a distanza di un decennio da Editions "La Guerre", in un numero di esemplari limitato, rilegati in volume, con un'introduzione firmata dall'autore e una prefazione di Paul Marguerite. L'unico esemplare di questa pubblicazione che conosciamo nella sua completezza è conservato al Palais du Roure di Avignone. Le opere vi risultano suddivise in quattro album, contenenti rispettivamente undici, dieci, undici e dieci incisioni. Non sappiamo come De Groux fosse riuscito a eludere il controllo della censura. Una possibile spiegazione è forse rintracciabile nel titolo «depistante» Le Visage de la Victoire, interpretato probabilmente dalla censura, che non sempre andava troppo per il sottile, come uno dei frequenti tributi retorici alla Vittoria [Morganti 2011].

\section{Henry de Groux, Le Visage de la Victoire: il racconto della guerra dai suoi prodromi}

Alcune fondamentali riflessioni esposte dall'artista nell'introduzione della raccolta rivelano la lucidità con cui osservava i fatti della guerra:

Ce qui frappe surtout dans cette guerre, c'est son opulente somptuosité d'horreur parfaite, son apocalyptique étrangeté, sa fureur, et, aggravant tout, son indéniable et colossale absurdité de machine fonctionnant à vide, précipitée par le mécanisme de son entraînement, de son impulsion, de son irréductible force, non moins que 
l'indéchiffrable complexité de ses vrais mobiles![...]'.

Poco più avanti, le sue parole disegnano la barbarie dei combattimenti come fatto non accessorio ma inevitabile e ineliminabile, perché appartenente alla natura dell'uomo:

L'auteur de ce recueil [...] confesse volontiers que sa surprise fut plus exactement de ne point les avoir vu survenir très antérieurement, après tant de signes et d'irrecusables prodromes. La guerre, comme de nombreux sages confirment, comme De Maistre, etc, est plutôt le véritable état moral de la vie. La paix, seule, serait insolite. La guerre fut de tout temps regardée comme l'état fondamental de l'existence des peuples, comme de celles des individus, la condition de leur santé et de

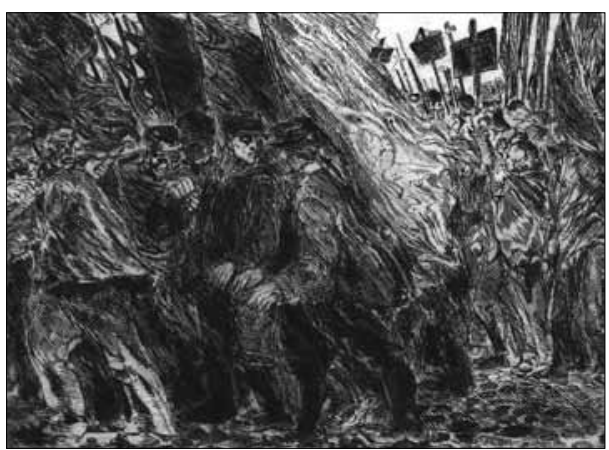
leur prestige 2 .

La sua disamina degli eventi bellici prende avvio nelle opere poste a corredo della prefazione. Alcune di queste, come l'acquaforte dal titolo Les drapeaux e la testatina priva di titolo, vanno a rievocare l'atmosfera euforica che caratterizzava la fase iniziale della mobilitazione.

H. De Groux, Les drapeaux, acquaforte

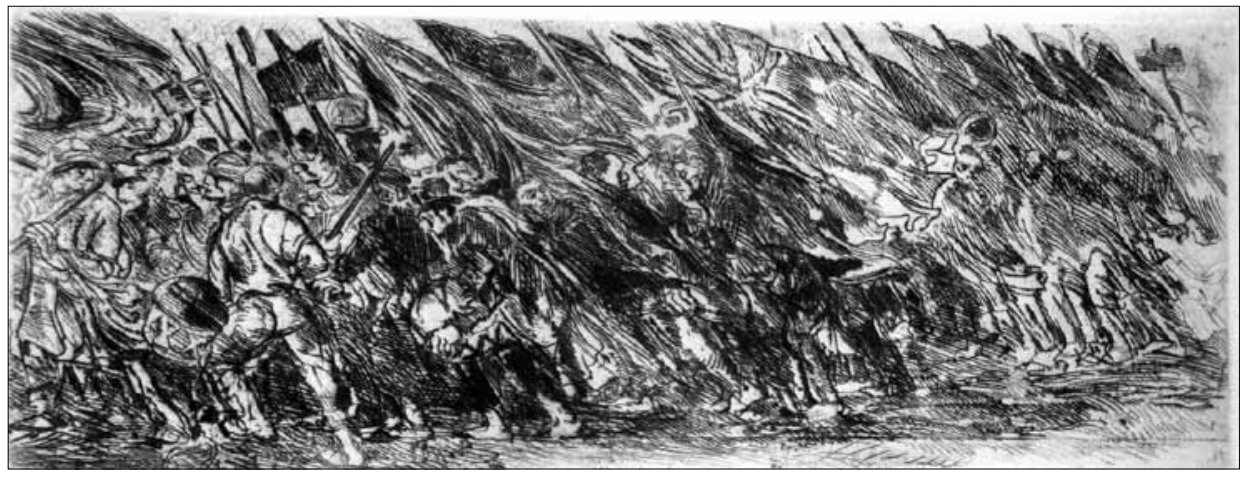

H. De Groux, Senza titolo (testatina), acquaforte

\footnotetext{
1 «Ciò che colpisce di questa guerra è la sua opulenta sontuosità di orrore perfetto, la sua innegabile e colossale assurdità di macchina funzionante a vuoto, precipitata dal meccanismo del suo trascinamento, dal suo impulso, dalla sua irriducibile forza, non meno che dall'indecifrabile complessità dei suoi veri motori» [De Groux 1924-1926: 2].

2 «L'autore di questa raccolta [...] confessa che la sua sorpresa sta nel fatto di non avere visto sopraggiungere prima queste tragiche circostanze, dopo tanti segni e irrecusabili prodromi. La guerra, come molti saggi confermano, come De Maistre, ecc., è piuttosto la reale condizione della vita. La pace, da sola, sarebbe insolita. La guerra è esistita in tutte le epoche, considerata come la fondamentale condizione morale della vita degli uomini, come di quella degli individui: la condizione della loro salute e del loro prestigio» [De Groux 1924-1926: 3].
} 


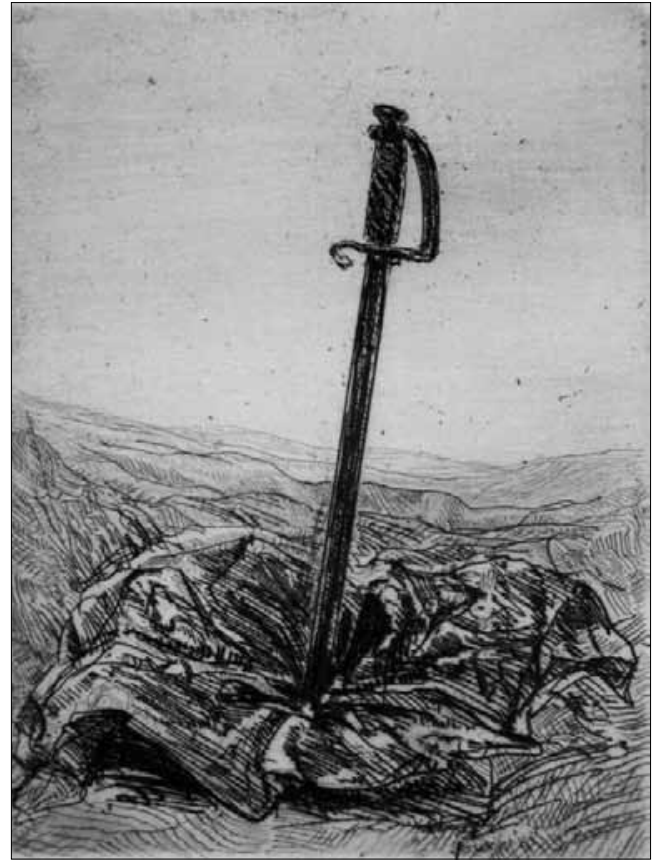

H. De Groux, Senza titolo (finalino), acquaforte

Sono lavori che, andando a evidenziare tanto il ruolo dei singoli (intellettuali sostenitori dell'interventismo) quanto i comportamenti collettivi di masse in preda all'esaltazione, mettono a nudo i perversi meccanismi che alimentavano la macchina bellica. Anche il finalino della prefazione, privo di titolo, presenta un soggetto alquanto emblematico: in un ambiente desolato e inospitale, che allude alla spiritualità disertata dall'umanità, travolta da istinti bellicosi e sanguinari, una spada è conficcata in un drappo steso al suolo. Se in essa si può riconoscere il simbolo classico della forza che inchioda a terra uno stendardo nemico (la virtù cardinale della fortezza è tradizionalmente associata alla spada), la forma dell'arma potrebbe al contempo suggerire un rimando all'iconografia della croce. In questo senso, assurgendo a un valore universale, la spada verrebbe a rappresentare lo strumento della sofferenza inferta da una guerra le cui armi di distruzione apportavano immani devastazioni anche all'ambiente, al corpo stesso della madre terra.

\section{La mostruosità bellica e la dimensione industriale della morte}

Nelle raffigurazioni di De Groux, la guerra si presenta anzitutto nella veste dell'orrore e della morte. Intesa come condizione naturale dell'esistenza umana, è evento folle e insensato, privo di qualsiasi finalità, per i vinti come per i vincitori. Altro non appare, insomma, che una «colossale macchina» che stritola gli uomini nei suoi orribili ingranaggi, all'interno della quale non è neppure possibile discernere fra buoni e cattivi, tra giusti e colpevoli. In questo processo, l'uomo, trasformato dalla tecnologia bellica, perde i suoi connotati di umanità. Emblematiche a tale proposito sono le immagini che De Groux dedica alla nuova arma del gas, ritraendo in particolare i soldati con le maschere antigas. Opere queste che, assurgendo a vere icone della spersonalizzazione e della ferocia dell'uomo, 


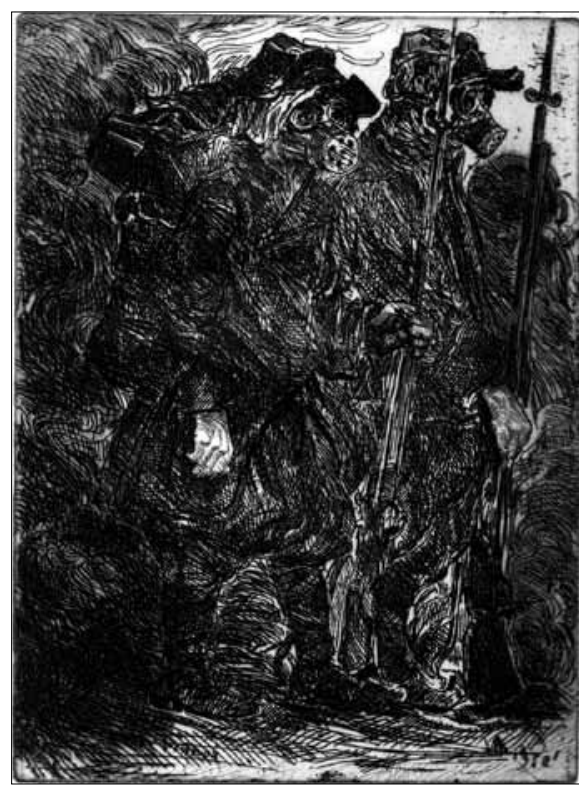

H. De Groux, Chevaliers errants, acquaforte

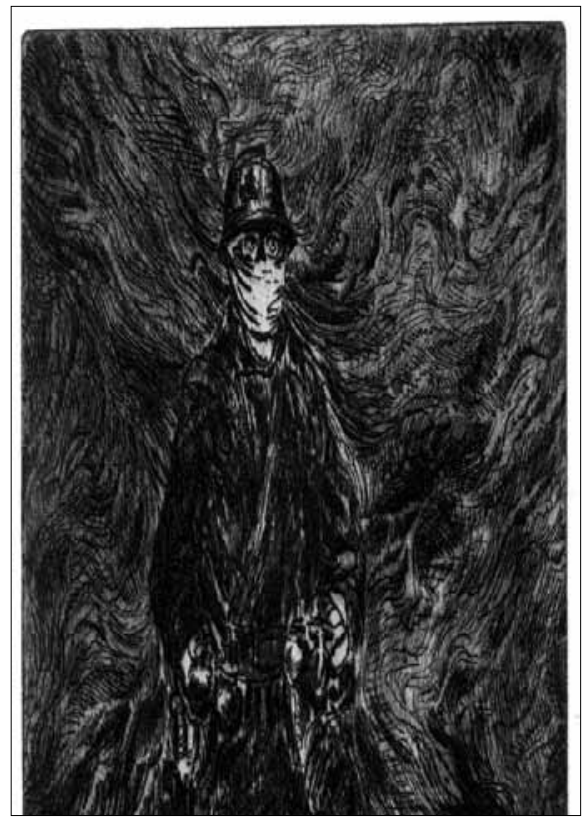

H. De Groux, Lanceur de granades, acquaforte

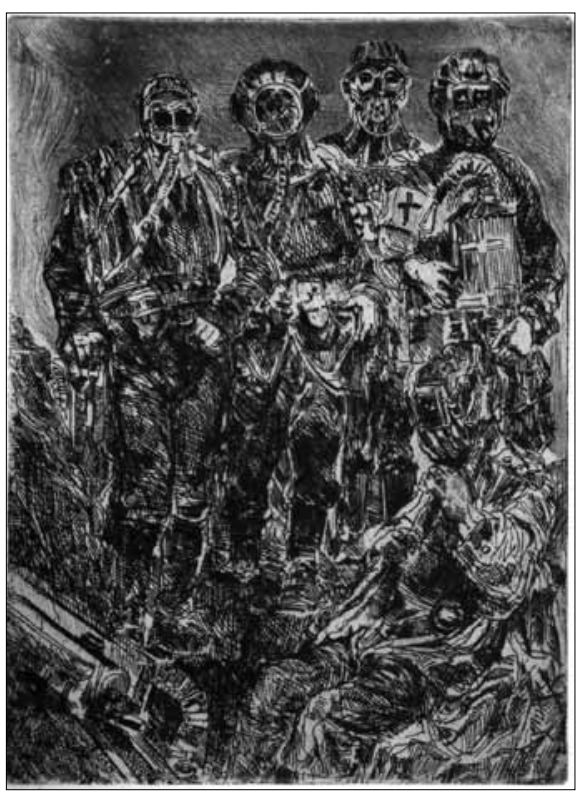

H. De Groux, Soldats masquées, acquaforte

vanno a delineare uno straordinario e del tutto inedito tema iconografico. Tali raffigurazioni fanno la loro apparizione nel terzo album de Le Visage de la Victoire, nelle opere Chevaliers errants e Soldats masquées, per trovare poi nel quarto, in Lanceur de granades e Assault de grenadiers, le realizzazioni più innovative, connotate da una notevole espressività e un potente dinamismo.

Il carattere folle e insensato che De Groux attribuisce a questi uomini-macchina è un modo per demistificare le immagini con cui la propaganda esaltava le moderne tecnologie belliche.

In ogni caso, come abbiamo accennato, il motivo portante de Le Visage de la Victoire è la raffigurazione della dimensione industriale della morte. Si impone infatti fin dal primo album (L'aube, Répression, L'invocation, Réveil au charnier), per proseguire nel secondo (L'hécatombe, L'autre tranchée, Soldat endormi) e 


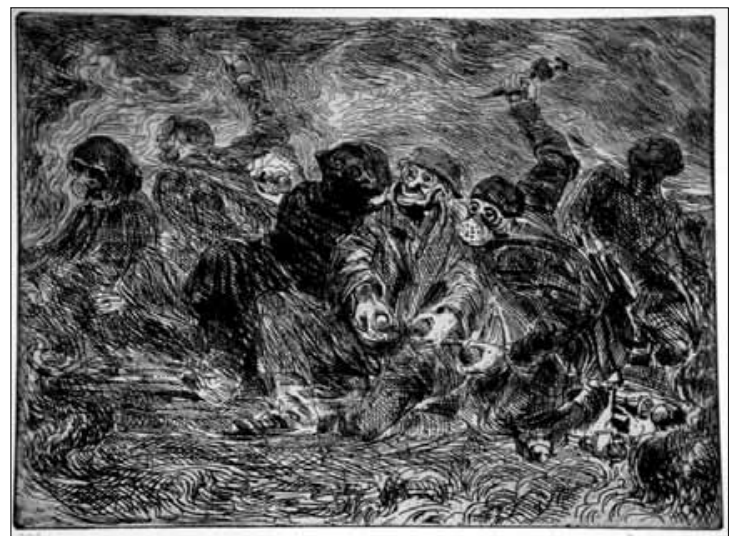

H. De Groux, Assault de grenadiers, acquaforte nel terzo (L'équipe funèbre), fino agli approdi del quarto album (Lux perpetua luceat eis, Dies irae e Retour au foyer), espressioni di un'arte originalissima che travalica i confini del tempo. In De Groux, tuttavia, la rappresentazione diretta dell'orrore non supera mai la soglia della ripugnanza e del ribrezzo. Il maestro non esibisce mai l'abominevole marciume dei corpi, lo stadio della

putrefazione dei cadaveri, non arriva mai, in altre parole, agli estremi raggiunti sovente da un Otto Dix, o da un Beckmann. Ricompone invece la realtà bruta della guerra con mano pietosa. A guidarlo non è l'intento di rendere quella realtà meno spaventosa, piuttosto un profondo rispetto per la dignità della persona. Si osservino in proposito la moltitudine di cadaveri allineati all'interno di una campagna dai confini indefiniti in Répression in cui i morti parrebbero dei civili: uomini privi di divise, elmi e armi, e donne con il foulard o la cuffia. O l'ammucchiarsi dei cadaveri che prolifera all'infinito, occupando gran parte dello spazio prospettico in L'hécatombe. O ancora il giovane soldato morto in Soldat endor$m i$ che dà l'impressione di dormire placidamente, similmente a un feto che riposi al sicuro nel

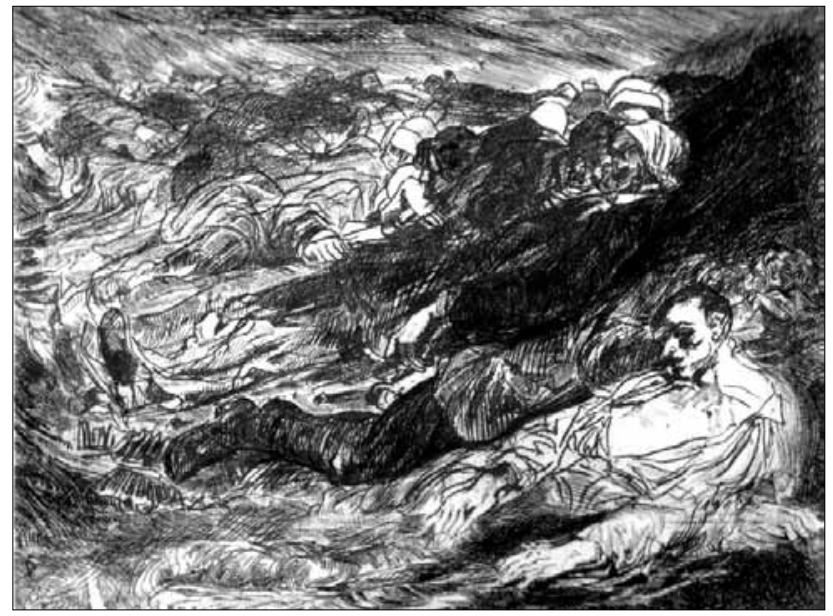

H. De Groux, Répression, acquaforte corpo della madre. 


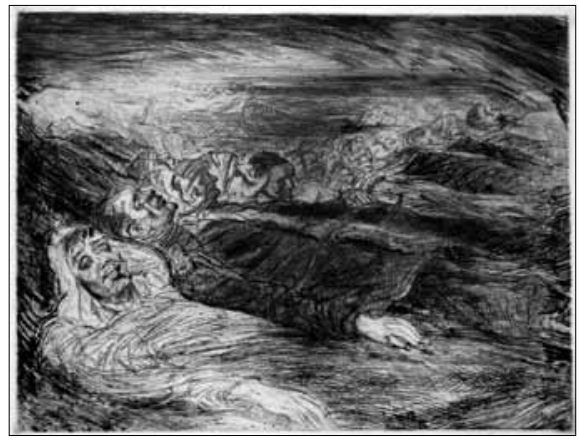

H. De Groux, L'hécatombe, acquaforte

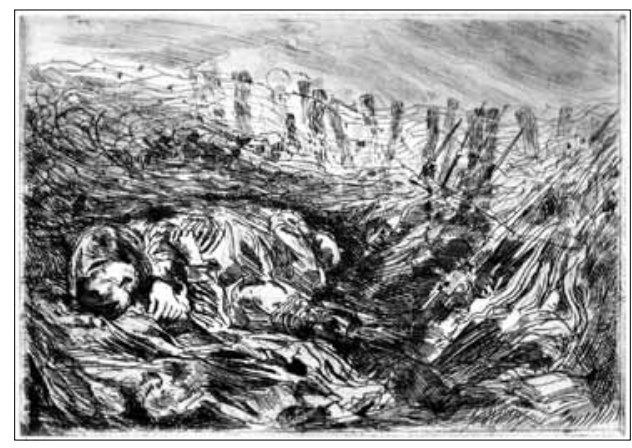

H. De Groux, Soldat endormi, acquaforte

\section{La denuncia della guerra negli occhi dei civili}

La denuncia delle atrocità della guerra trova nella raccolta Le Visage de la Victoire dei momenti di grande intensità e spessore umano, in particolare nelle opere

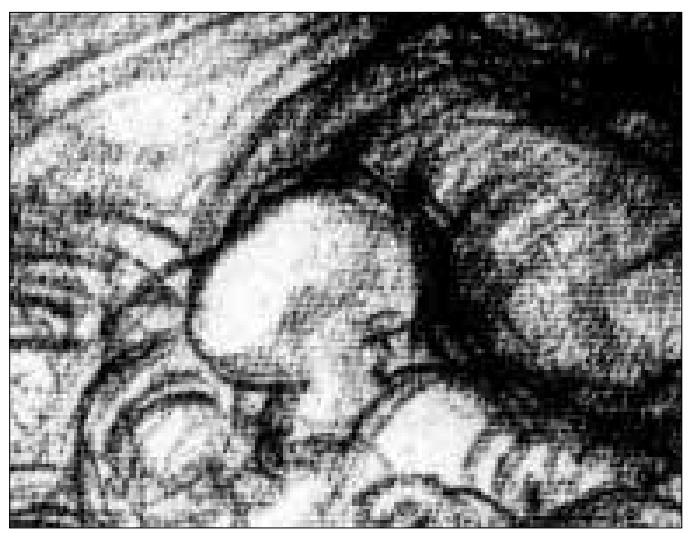

H. De Groux, Les innocents, vernice molle in cui le vittime sono degli innocenti. In Les innocents lo sguardo dell'artista va a cogliere un'atroce tragedia che ha colpito dei civili.

I riferimenti incisi in lastra indicano che la scena è ambientata a Parigi, nel 1915. L'opera, realizzata con tratti rapidi e scarni, mostra la disperazione di una madre tra i cadaveri dei figli, travolti dal crollo della loro abitazione. Nel turbinare convulso dei segni, uno

squarcio di luce è diretto verso questa grandiosa figura di donna il cui urlo impotente rappresenta un severo monito all'umana follia. Di grande forza espressiva è la scena raffigurata in Réfugiés, eseguita a vernice molle. Qui una madre stringe attorno a sé i suoi tre figlioli, cercando di dare loro conforto. Gli occhi infossati, gli sguardi impauriti e invocanti aiu-

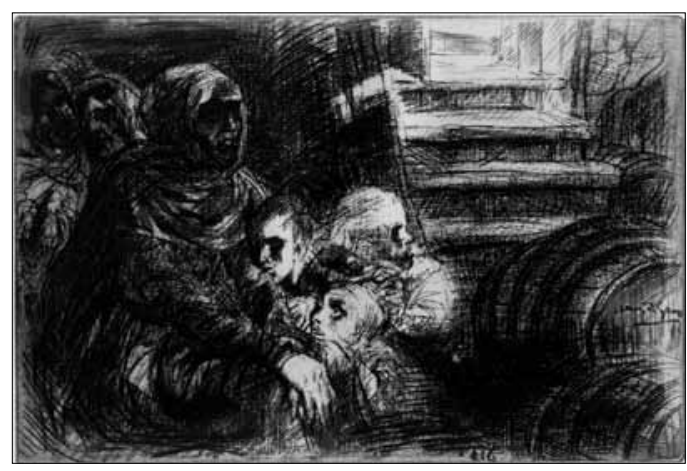

H. De Groux, Réfugiés, vernice molle 
to dei piccoli rappresentano una chiara denuncia delle sofferenze imposte ai più deboli, già provati dalla scarsità di cibo e dalla lontananza dei capofamiglia impegnati a combattere al fronte.

\section{Il volto del nemico-prigioniero}

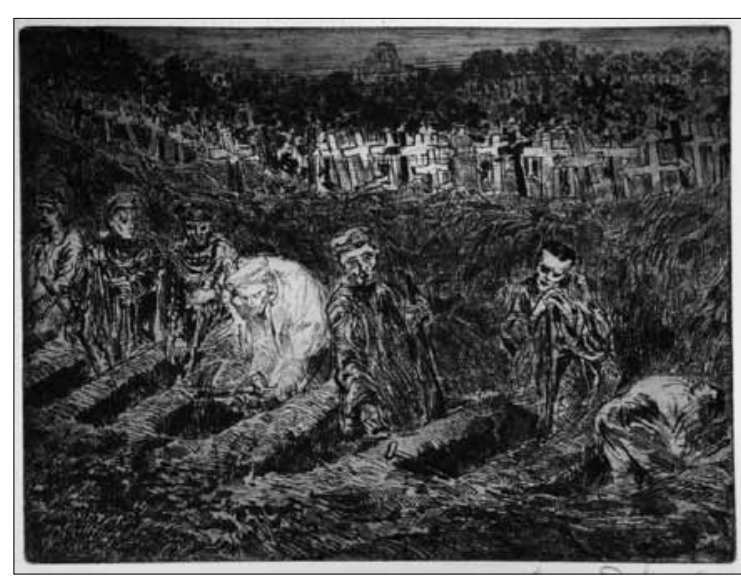

H. De Groux, Prisonniers fossoyeurs, acquaforte
A far da contrappeso alle molte immagini di ferocia, un certo numero di stampe della raccolta sono dedicate all'iconografia del prigioniero. In esse l'artista esplora l'umanità del nemico ridotto in cattività, spogliato da ogni strumento di aggressività e di potenza. In qualche caso si tratta di scene di gruppo, in altri di veri e propri ritratti che delineano con fine sen-

sibilità i più lievi moti dell'animo: la sofferenza, il timore e l'umiliazione dei reclusi. Marcati aspetti emozionali emergono nelle raffigurazioni di prigionieri intenti a scavare delle sepolture, i cosiddetti «prisonniers fossoyeurs», cui l'artista attribuisce il compito di mostrare allegoricamente la precarietà e l'effimero insito nell'esistenza dei soldati.

Questa tematica raggiunge toni di grande intensità in opere come Prisonniers fossoyeurs dove la presenza di numerose buche vuote, in macabra attesa di un ospite, sottolineano con grande efficacia l'immane entità della strage prodotta dai combattimenti. I «prigionieri scavatori» non appaiono

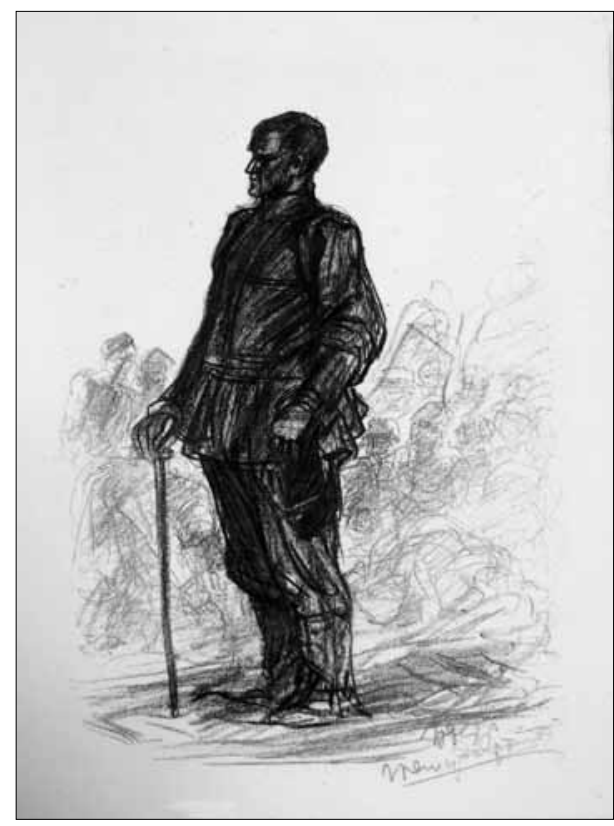

H. De Groux, Prisonnier fossoyeur, vernice molle 


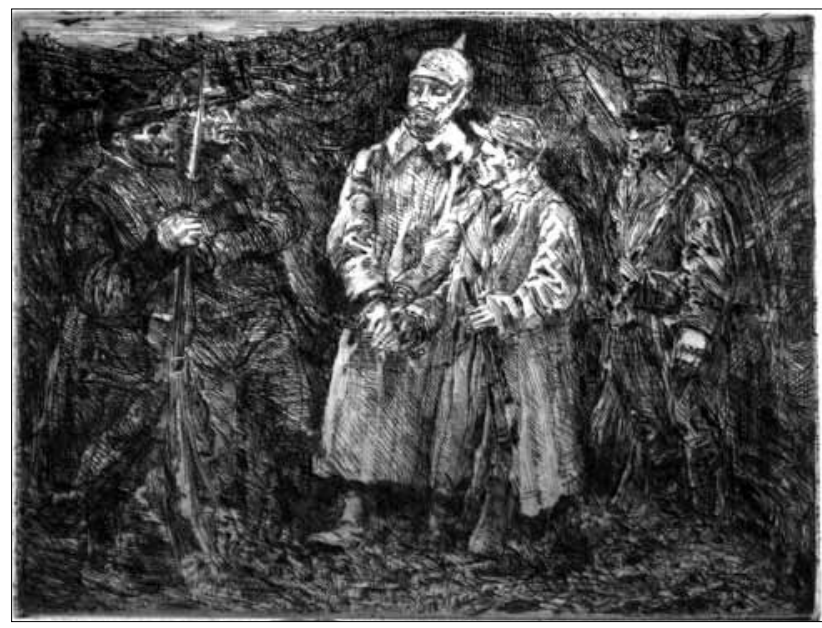

H. De Groux, Premier captif, acquaforte mai dei nemici da schernire e disprezzare, ma piuttosto degli uomini le cui sofferenze possono suscitare commozione e pietà.

Assai significative in questo senso sono le immagini nelle quali il nemico in cattività è mostrato in primo piano. Molto intensa, ad esempio, è la figura di Prisonnier fossoyeur, ripreso in controluce con fare pensieroso.

Sullo sfondo della stampa sono tratteggiate delle sagome poco definite, la cui presenza sembra acuire l'assoluto isolamento del personaggio principale. Quale precipuo valore attribuisse l'artista a una tale raffigurazione fatta di nulla è difficile dirlo. L'opera sembra comunque riflettere sulla difficile condizione psicologica del prigioniero.

Il prigioniero rimane per De Groux meritevole di pietà e di considerazione anche quando appartiene alle più alte gerarchie militari, come l'ufficiale tedesco rappresentato nell'acquaforte Premier captif. Osservato con incredulità e forse con pietà dai soldati francesi che l'attorniano, il militare si erge orgogliosamente in tutta la sua altezza, tenendo però gli occhi serrati, segno della riluttanza con cui si rassegna al disonore della cattura. Una luce sovrannaturale sembra ingigantirne la figura, quasi a rendere onore al valore del soldato nemico.

Emil Baumann legge però la scena in modo completamente differente: «Un soldato francese, vigile e beffardo, conduce la sua preda, un sergente rigido e baffuto che abbassa gli occhi, mortificato dalla sconfitta e senza comprenderla. Gli altri militari che lo fissano brutalmente sono più ridicoli che epici. L'insieme è ripreso da uno schizzo dal vivo, ma raggiunge un'ampiezza di significati imprevisti, il contrasto tra due umanità ostili e che non giungono mai a intendersi» [Baumann 1936, 244]. 


\section{Conclusioni}

Una linea ideale riallaccia la raccolta Le Visage de la Victoire ai Disastri della guerra di Goya, cui l'accomuna lo spirito polemico, la truce visione somigliante a un incubo, l'assenza di ogni indugio nella cronaca e nella mera documentazione. Impossibile, poi, sfuggire il confronto con la raccolta di incisioni Der Krieg di Otto Dix. Nonostante il differente approccio tra i due maestri, espressionista Dix, visionario-simbolista De Groux, in entrambi colpisce la capacità di ricercare il senso profondo degli eventi e di comunicare un messaggio antimilitarista che sovrasta il proprio tempo.

\section{Bibliografia}

Baumann E. 1936, La vie terrible d'Henry de Groux, Paris: Grasset.

De Groux H. 1924-1926, Le Visage de la Victoire, Paris: Genèse de ce livre.

Morganti C. 2011, Gli orrori della guerra nell'opera grafica di Henry De Groux, "Grafica d'Arte", 90.

Morganti C. 2013, Gli orrori della Grande Guerra nelle incisioni di un geniale artista simbolista. Henry De Groux, Le Visage de la Victoire, Tricase: Libellula.

\section{Risorse}

Rivista on line ArteGrandeGuerra http://www.artegrandeguerra.it/p/rivista.html

Intervista recensione a Carol Morganti su Gli orrori della Grande Guerra nelle incisioni di un geniale artista simbolista. Henry De Groux. Le Visage de la Victoire http://www.artegrandeguerra.it/2013/10/henry-de-groux-le-visage-de-la-victoire.html 